\title{
Is partial nephrectomy worth performing compared to radical nephrectomy for small, localised renal cortical tumours in geriatric patients?
}

Jing Kai Jackie ${\underline{\text { am }^{1}}}^{1}$, MBBS, Sher Yin $\underline{\operatorname{Tan}}^{1}$, mBBS, Kian Tai $\underline{\text { Chong}}{ }^{2}$, MBBS

INTRODUCTION This study aimed to evaluate the likelihood of progression to chronic kidney disease (CKD) in a cohort of geriatric Asian patients who underwent either partial nephrectomy (PN) or radical nephrectomy (RN) for localised pT1 kidney tumours.

METHODS Geriatric patients aged > 65 years who underwent PN or RN from 2005 to 2014 for localised kidney masses $<7 \mathrm{~cm}$ at a single institution were reviewed retrospectively. Preoperative and postoperative estimated glomerular filtration rates (eGFRs) were calculated using the Modification of Diet in Renal Disease Study equation. Clinicopathological data was analysed for new-onset CKD after at least five years of follow-up. Postoperative complications were measured using the modified Clavien classification system.

RESULTS Among 67 patients, 36.4\% had diabetes mellitus and $80.3 \%$ had hypertension. Pre-existing CKD with eGFR $\leq 60 \mathrm{~mL} / \mathrm{min} / 1.73 \mathrm{~m}^{2}$ was present in $23.3 \%$ of PN and $43.2 \%$ of RN patients. RN and PN patients had similar postoperative morbidity. Those who underwent PN had immediate decline followed by gradual improvement of kidney function at six months after surgery. This improvement was sustained even for patients with four years of clinical follow-up. Among patients with normal preoperative kidney function, new-onset CKD after surgery was found in $20.0 \%$ and $52.9 \%$ of PN and RN patients, respectively $(p=0.007)$.

CONCLUSION In our study, geriatric patients who underwent PN for localised kidney mass $<7 \mathrm{~cm}$ had immediate decline but gradual and sustained improvement in kidney function. PN also resulted in less new-onset CKD through a five-year follow-up period.

Keywords: overall survival, partial nephrectomy, radical nephrectomy, renal cortical cancer

\section{INTRODUCTION}

To our knowledge, in the only prospective randomised trial to evaluate oncological outcomes between patients with partial nephrectomy $(\mathrm{PN})$ and radical nephrectomy $(\mathrm{RN})$ for low-stage renal cell carcinoma, the EORTC (European Organization for Research and Treatment of Cancer) Trial 30904 showed no difference in overall survival. ${ }^{(1)}$ As for postoperative renal function, $\mathrm{PN}$ has been shown to reduce the risk of moderate chronic kidney disease (CKD), assuming normal preoperative kidney function. ${ }^{(2)}$ Taking reference from these two studies, we wanted to investigate whether it was still worthwhile to perform nephron-saving PN for geriatric patients in Singapore.

In our study, geriatric patients were defined as individuals aged over 65 years. Geriatric patients are increasingly common in daily clinical practice, as Singapore faces an ageing population with rising life expectancy levels (mean age 82 years in 2013). ${ }^{(3)}$ The widespread use of cross-sectional imaging has also led to increased incidence of incidentally diagnosed renal tumours among elderly patients.

Current practice guidelines recommend $\mathrm{PN}$ for the treatment of cT1a small renal cortical tumours whenever it is technically feasible, as nephron preservation has been shown to prevent the sequelae of CKD. This includes complications of anaemia, malnutrition, neuropathy, reduced quality of life, and increased risk of cardiovascular disease and mortality. However, the randomised trial from the EORTC showed that there was no difference in oncological outcomes between patients undergoing $\mathrm{PN}$ and $\mathrm{RN}$, and the question of whether it was still worthwhile to perform $\mathrm{PN}$ for our geriatric patients assumed significance.

In clinical practice, the decision regarding treatment modality is based on thoughtful consideration of the balance to be achieved between preservation of renal function and oncologic efficacy. Our goal was thus to evaluate long-term renal and oncological outcomes for treating T1-stage renal cortical tumours among patients aged over 65 years.

\section{METHODS}

We retrospectively analysed data for 67 patients who underwent surgery for small renal cortical tumours at a single institution in Singapore between 2005 and 2014, to achieve a minimum follow-up duration of two years.

The study received ethics approval from the institutional review board (National Healthcare Group Domain Specific Review Board ref 2009/00939). After ethics approval, patients who were eligible were selected for review based on the following criteria: (a) aged $>65$ years at time of diagnosis; (b) macroscopic tumour size $<7 \mathrm{~cm}$; (c) had undergone either PN or RN; and (d) had organ-confined tumours with no lymph node involvement or distant metastasis.

Patients excluded were those: (a) with repeat PN or RN for recurrence; (b) with only one functioning kidney; (c) without any 
creatinine level measurements performed at intervals of six months, one year and two years postoperatively; and (d) who had undergone radiofrequency ablation as the main therapeutic procedure. The date of the last data analysis was September 2016. Patients with metastatic renal cell carcinoma were also excluded from data analysis.

The parameters analysed included preoperative patient factors, including age, ethnicity, gender, comorbidities (e.g. diabetes mellitus, last glycated haemoglobin level, hypertension, presence of ischaemic heart disease, ASA [American Society of Anesthesiologists] score) and haemoglobin levels. Information on tumour factors, such as tumour histological differentiation and pathological staging, was also collected. Pathological staging was performed based on the American Joint Committee on Cancer classification system, seventh edition, and histological differentiation was graded according to the Fuhrman nuclear grading system. Renal factors, such as estimated glomerular filtration rate (eGFR) measurements, were estimated using the Modification of Diet in Renal Disease (MDRD) and Chronic Kidney Disease Epidemiology Collaboration (CKD-EPI) equation, ${ }^{(4)}$ for which creatinine levels were measured preoperatively, immediately after surgery, and during follow-up at six months, one year, two years, three years, four years and five years. Intraoperative characteristics such as warm ischaemia time, cold ischaemia time, total surgical time, and the presence of intraoperative and postoperative complications were also taken into account. Postoperative complications were classified using the modified Clavien grading.

Due to the small number of patients in each individual subgroup, results generated from any multivariate logistic regression analysis were unlikely to be statistically significant. Hence, univariate logistic regression analysis was performed based on patients' MDRD scores and plotted over a five-year period.

The main aim was to assess postoperative kidney function, for which eGFR was first recorded postoperatively and subsequently at six months, one year, two years, three years, four years and five years during follow-up outpatient visits. We also took into account any new-onset CKD, as defined by the occurrence of eGFR $\leq 60 \mathrm{~mL} / \mathrm{min} / 1.73 \mathrm{~m}^{2}$ on two consecutive follow-up visits of at least three months apart or by end-stage renal failure, which was defined as eGFR $<15 \mathrm{~mL} / \mathrm{min} / 1.73 \mathrm{~m}^{2}$. Besides these, overall survival, cancer-specific survival and progression-free survival were ascertained by recording patients' death status, presence of recurrence and cause of death.

\section{RESULTS}

Table I details the general demographics of our patient population in both the PN and RN groups. RN patients were associated with increased T-staging (RN 67.6\%, PN 10.0\%; $\mathrm{p}<0.001$ ) and higher incidence of new-onset CKD or renal failure (RN 52.9\%, PN 20.0\%; $p=0.007$ ) when compared to PN patients. At baseline, a larger proportion of RN patients had preoperative CKD ( $R N$ 43.2\%, PN 23.3\%) in comparison with PN patients. Patients in the RN group also had more pre-existing comorbidities, such as diabetes mellitus (RN 44.4\%, PN 26.7\%) and hypertension (RN 83.3\%, PN 76.7\%). Postoperative complications were similar in both $\mathrm{RN}$ and $\mathrm{PN}$ patients (PN 32.1\%, RN 33.3\%).
Of 67 patients included in the overall survival analysis, 32 patients had creatinine measurements at follow-up visits of up to five years. However, 8 (11.9\%) patients died before completing five years of follow-up, 20 (29.9\%) patients were diagnosed after 2011 and hence did not have five years of follow-up data in September 2016, and 7 (10.4\%) patients were lost to follow-up for other reasons.

Table II shows that average preoperative MDRD values for $\mathrm{RN}$ and $\mathrm{PN}$ patients were $65.8 \pm 33.0 \mathrm{~mL} / \mathrm{min} / 1.73 \mathrm{~m}^{2}$ and 78.7 $\pm 23.2 \mathrm{~mL} / \mathrm{min} / 1.73 \mathrm{~m}^{2}$, respectively, confirming the general consensus that patients undergoing $\mathrm{RN}$ have poorer baseline renal function. Average MDRD values for RN and PN patients at the fiveyear follow-up dropped to $42.9 \pm 20.5 \mathrm{~mL} / \mathrm{min} / 1.73 \mathrm{~m}^{2}(p<0.001)$ and $64.1 \pm 19.7 \mathrm{~mL} / \mathrm{min} / 1.73 \mathrm{~m}^{2}(p=0.019)$, respectively, corresponding to a $35.0 \%$ and $18.0 \%$ decrease in MDRD scores for RN and PN, respectively. At the five-year follow-up, $46.7 \%$ of patients who underwent PN had CKD $(p=0.625)$ as compared to $70.6 \%$ of patients in the RN group ( $p=0.125$ ).

Fig. 1a shows the average MDRD values of all 67 patients across the five-year follow-up period. Both patients from $\mathrm{RN}$ and $\mathrm{PN}$ groups experienced an immediate steep decline of renal function postoperatively, with $\mathrm{PN}$ patients showing improved renal function at the six-month follow-up. This improvement was sustained throughout the five-year follow-up period as MDRD values plateaued with time. Comparatively, $\mathrm{RN}$ patients experienced a further decline in renal function at six months postoperatively, with no significant improvement in renal function throughout the five-year follow-up period.

To further improve the accuracy of our findings regarding the renal function of $\mathrm{RN}$ and $\mathrm{PN}$ patients, we further narrowed this analysis to patients who had at least four years of follow-up data (Fig. 1b). A similar trend was seen among these patients $(n=37)$ as for all 67 patients, emphasising the accuracy of observations in Fig. 1a.

\section{DISCUSSION}

To make it easier to identify patients with CKD, equations such as MDRD and CKD-EPI were formulated to estimate eGFR. For our study, we decided to use the MDRD equation, based on the findings of Masson et al. ${ }^{(5)}$ In this earlier study, 825 patients were monitored for their creatinine and insulin clearance levels. Results showed that for patients with eGFR $<60 \mathrm{~mL} / \mathrm{min} / 1.73 \mathrm{~m}^{2}$ and in the range $60-90 \mathrm{~mL} / \mathrm{min} / 1.73 \mathrm{~m}^{2}$, MDRD showed a significantly higher accuracy of $80 \%$ when compared to an accuracy of $74 \%$ when CKD-EPI was used. For eGFR $>90 \mathrm{~mL} / \mathrm{min} / 1.73 \mathrm{~m}^{2}$, there were no significant differences between the two equations in terms of performance. As our study focused on renal function in a Singapore geriatric population, we expected many of our patients to have pre-existing renal disease and therefore assumed that the MDRD equation would be more applicable for data analysis.

The general prevalence of diabetes mellitus and hypertension in Singapore in 2010 was $11.3 \%$ and $23.5 \%$, respectively. ${ }^{(6)}$ We found that $34.3 \%$ of our patients had pre-existing CKD prior to undergoing $\mathrm{RN}$ and $\mathrm{PN}$. It is typical for the geriatric population to have more comorbidities when compared to the general population. Accordingly, in our study, $36.4 \%$ and $80.3 \%$ of patients had diabetes mellitus and hypertension, respectively, at the time of their renal 
Table I. Demographics and baseline clinical features of patients.

\begin{tabular}{|c|c|c|c|c|}
\hline \multirow[t]{2}{*}{ Variable } & \multicolumn{3}{|c|}{ No. (\%) } & \multirow[t]{2}{*}{ p-value } \\
\hline & Total $(n=67)$ & $P N(n=30)$ & RN $(n=37)$ & \\
\hline Gender & & & & 0.953 \\
\hline Female & $31(46.3)$ & $14(46.7)$ & $17(45.9)$ & \\
\hline Male & $36(53.7)$ & $16(53.3)$ & $20(54.1)$ & \\
\hline Age* $^{*}(y r)$ & $70(67-74)$ & $70(67-71)$ & $71(67-75)$ & 0.0724 \\
\hline Ethnicity & & & & $<1.000$ \\
\hline Chinese & $66(98.5)$ & $30(100.0)$ & $36(97.3)$ & \\
\hline Malay & $1(1.5)$ & $0(0)$ & $1(2.7)$ & \\
\hline Diabetes mellitus & & & & 0.135 \\
\hline Yes & $24(36.4)$ & $8(26.7)$ & $16(44.4)$ & \\
\hline No & $42(63.6)$ & $22(73.3)$ & $20(55.6)$ & \\
\hline Hypertension & & & & 0.498 \\
\hline Yes & $53(80.3)$ & $23(76.7)$ & $30(83.3)$ & \\
\hline No & $13(19.7)$ & $7(23.3)$ & $6(16.7)$ & \\
\hline CKD & & & & 0.152 \\
\hline Yes & $21(32.3)$ & $7(23.3)$ & $14(40.0)$ & \\
\hline No & $44(67.7)$ & $23(76.7)$ & $21(60.0)$ & \\
\hline Staging of CKD & & & & 0.088 \\
\hline Yes & $23(34.3)$ & $7(23.3)$ & $16(43.2)$ & \\
\hline No & $44(65.7)$ & $23(76.7)$ & $21(56.8)$ & \\
\hline Estimated renal function ${ }^{t, \neq}\left(\mathrm{mL} / \mathrm{min} / 1.73 \mathrm{~m}^{2}\right)$ & $71.8 \pm 29.4$ & $78.7 \pm 23.2$ & $65.8 \pm 33.0$ & 0.0775 \\
\hline ASA level & & & & 0.619 \\
\hline 2 & $33(61.1)$ & $15(57.7)$ & $18(64.3)$ & \\
\hline 3 & $21(38.9)$ & $11(42.3)$ & $10(35.7)$ & \\
\hline T-staging & & & & $<0.001$ \\
\hline $1 \mathrm{a}$ & $39(58.2)$ & $27(90.0)$ & $12(32.4)$ & \\
\hline $1 \mathrm{~b}$ and above & $28(41.8)$ & $3(10.0)$ & $25(67.6)$ & \\
\hline Postoperative complications & & & & 0.923 \\
\hline Yes & $19(32.8)$ & $9(32.1)$ & $10(33.3)$ & \\
\hline No & $39(67.2)$ & $19(67.9)$ & $20(66.7)$ & \\
\hline New-onset CKD or renal failure & & & & 0.007 \\
\hline Yes & $24(37.5)$ & $6(20.0)$ & $18(52.9)$ & \\
\hline No & $40(62.5)$ & $24(80.0)$ & $16(47.1)$ & \\
\hline
\end{tabular}

Percentages were calculated based on available data. Data presented as *median (interquartile range) and tmean \pm standard deviation. $\neq$ Estimated renal function was measured based on the Modification of Diet in Renal Disease Study equation. ASA: American Society of Anesthesiologists score; CKD: chronic kidney disease; PN: partial nephrectomy; RN: radical nephrectomy

Table II. Stage of CKD and renal function among patient groups.

\begin{tabular}{|c|c|c|c|c|c|}
\hline \multirow[t]{3}{*}{ Variable } & \multicolumn{4}{|c|}{ No. (\%) } & \multirow[t]{3}{*}{ p-value } \\
\hline & \multicolumn{2}{|c|}{ Baseline CKD } & \multicolumn{2}{|c|}{ CKD at 5 yr } & \\
\hline & Yes & No & Yes & No & \\
\hline \multicolumn{6}{|l|}{ Stage of CKD } \\
\hline Partial nephrectomy & $7(23.3)$ & $23(76.7)$ & $7(46.7)$ & $8(53.3)$ & 0.625 \\
\hline Radical nephrectomy & $16(43.2)$ & $21(56.8)$ & $12(70.6)$ & $5(29.4)$ & 0.125 \\
\hline \multicolumn{6}{|c|}{ Estimated renal function ${ }^{*,+}\left(\mathrm{mL} / \mathrm{min} / 1.73 \mathrm{~m}^{2}\right)$} \\
\hline Partial nephrectomy & $78.7 \pm 23.2$ & & $64.1 \pm 19.7$ & & 0.019 \\
\hline Radical nephrectomy & $65.8 \pm 33.0$ & & $42.9 \pm 20.5$ & & $<0.001$ \\
\hline
\end{tabular}

*Data presented as mean \pm standard deviation. †Estimated renal function was measured based on the Modification of Diet in Renal Disease Study equation. CKD: chronic kidney disease 

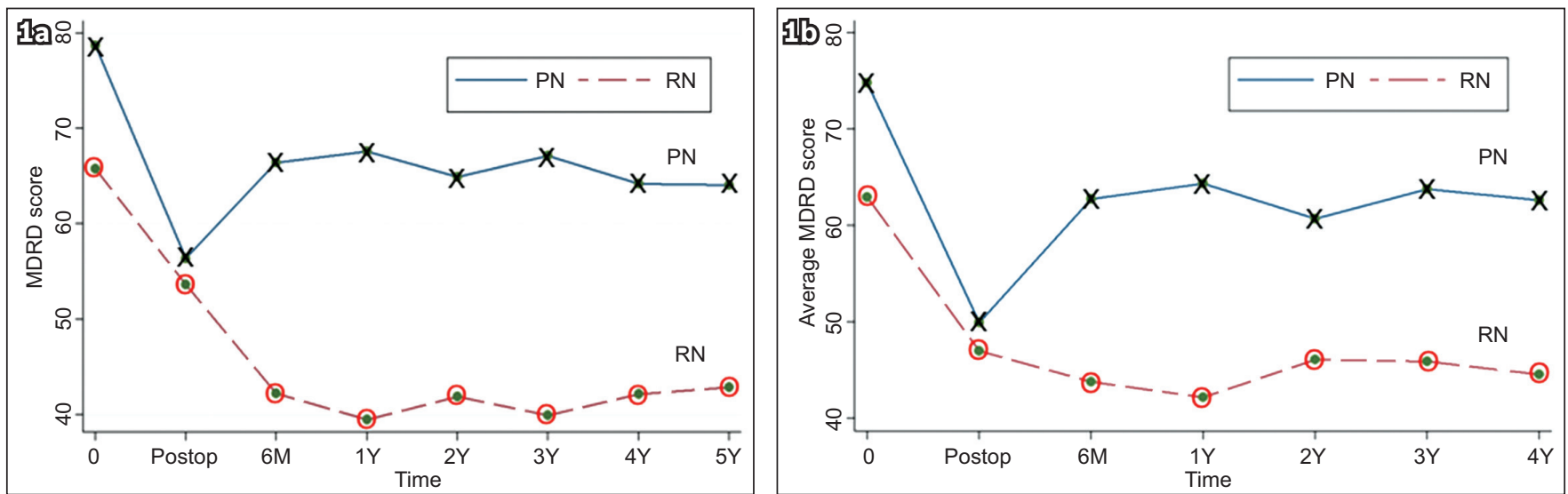

Fig. 1 Chart shows MDRD values of (a) all patients $(n=67)$ and $(b)$ patients with at least four years of follow-up data ( $n=37)$. M: months; MDRD: Modification of Diet in Renal Disease Study; PN: partial nephrectomy; Postop: post operation; RN: radical nephrectomy; Y: years

carcinoma diagnosis. This result has significantly altered our opinion of how small renal cortical tumours in the geriatric population should be managed, given that there is widespread evidence of nephropathy precipitated by diabetes mellitus and hypertension in older patients. ${ }^{(7)}$

A study by Mashni et $\mathrm{al}^{(8)}$ demonstrated that for patients with normal kidney function, $\mathrm{RN}$ was associated with a significantly higher risk for developing CKD and worse overall survival than $\mathrm{PN}$, implying that $\mathrm{PN}$ should be the treatment of choice for nephron-sparing purposes. These authors, however, provided no details about the preoperative renal function of the patients at the time of diagnosis, so that the likelihood of any patients having developed CKD prior to diagnosis of renal cancer remains unexplored and unknown. By considering the preoperative renal function of patients and narrowing the cohort size to only geriatric patients who inherently have more comorbidities, our study demonstrated that patients who underwent PN had an immediate decline in renal function after surgery followed by gradual improvement of kidney function by six months postoperatively, and that this improvement was sustained in patients even at the five-year follow-up visit. Comparatively, those who underwent RN showed an immediate decline in renal function after surgery that was followed by decline throughout the five years of follow-up.

Our study confirmed findings in the published literature that patients undergoing RN had significantly higher (43.2\%) rates of pre-existing CKD when compared to patients undergoing PN $(23.3 \%)$. This conforms with real-life clinical practice, where surgeons are likely to recommend $\mathrm{PN}$ to patients who have higher viable renal function at diagnosis.

Taking reference from the EORTC randomised trial, ${ }^{(1)}$ where there was no difference in the oncological outcomes of patients undergoing PN and RN, we aimed to evaluate and compare residual renal function between patients who had $\mathrm{RN}$ and those who had $\mathrm{PN}$, specifically among a Singapore geriatric patient population with small renal cortical tumours. We believed that our findings would allow urologists from Singapore to better decide whether RN or PN would be a more appropriate procedure for their patients.

Our present study had some limitations. We were unable to demonstrate any association between new-onset CKD and surgical intervention or the patient's pre-existing comorbidities even though a majority of patients in our study had nephropathic comorbidities, such as diabetes mellitus and hypertension. Our study was mainly focused on the development of new-onset CKD rather than giving a holistic view of the patients' overall survival. Given that our study had a non-randomised retrospective design and was performed at a single centre, there was significant inherent bias in patient selection, as our centre mainly offers $\mathrm{PN}$ to patients with good renal function and high life expectancy. It would also have been preferable if recurrence of disease in patients had been documented, to accurately weigh the comparative benefits and disadvantages of RN and PN.

In our study, geriatric patients who underwent PN for localised kidney mass $<7 \mathrm{~cm}$ had immediate decline in renal function after surgery but showed a gradual and sustained improvement in kidney function over the five-year follow-up period. PN patients had lower incidence of new-onset CKD, which was sustained throughout the five-year follow-up period. Our findings suggest that it is worthwhile to consider nephron-sparing PN for patients with small renal cortical tumours from the Singapore geriatric population, especially given that many of these patients have pre-existing comorbidities that may further worsen renal function following surgery. There is a need for further studies in this area so as to more accurately demonstrate the benefits and drawbacks of PN for the Singapore geriatric population.

\section{REFERENCES}

1. Van Poppel H, Da Pozzo L, Albrecht W, et al. A propective, randomized EORTC intergroup phase 3 study comparing the oncological outcome of elective nephron-sparing surgery and radical nephrectomy for low-stage renal cell carcinoma. Eur Urol 2011; 59:543-52.

2. Scosyrev E, Messing EM, Sylvester R, Campbell S, Van Poppel H. Renal function after nephron-sparing surgery versus radical nephrectomy: results from EORTC randomized trial 30904. Eur Urol 2014; 65:372-7.

3. Khalik S. Singapore ranks world No. 4 for life expectancy. In: The Straits Times [online]. Available at: https://www.straitstimes.com/singapore/singapore-ranksworld-no-4-for-life-expectancy. Accessed March 6, 2019.

4. Levey AS, Stevens LA, Schmid CH, et al; CKD-EPI (Chronic Kidney Disease Epidemiology Collaboration). A new equation to estimate glomerular filtration rate. Ann Intern Med 2009; 150:604-12.

5. Masson I, Flamant M, Maillard N, et al. MDRD versus CKD-EPI equation to estimate glomerular filtration rate in kidney transplant recipients. Transplantation 2013; 95:1211-7.

6. Singapore Heart Foundation. Risk Factors. Available at: http://www.myheart.org. sg/article/about-the-heart-and-heart-disease/statistics/risk-factors/77. Accessed September 3, 2018.

7. AKH Lim. Diabetic nephropathy - complications and treatment. Int J Nephrol Renovasc Dis 2014; 7:361-81.

8. Mashni JW, Assel M, Maschino A, et al. New chronic kidney disease and overall survival after nephrectomy for small renal cortical tumors. Urology 2015; 86:1137-43. 